\title{
A New Approach to Knowledge Acquisition by Repertory Grids
}

\author{
Sanjiv K. Bhatia and Qi Yao \\ Department of Mathematics \& Computer Science \\ University of Missouri - St. Louis \\ St. Louis, MO 63121-4499
}

\begin{abstract}
Personal construct theory is one of the important tools for knowledge acquisition. It provides an expert's opinion of the relationship between objects and their properties in the form of a repertory grid. The current techniques provide the repertory grid in the form of a matrix of integers. This paper describes the preliminary attempts for elicitation and analysis of repertory grid using a range of integers for each relationship. Such an elicitation leads to a more natural description of the expert's knowledge in addition to a better user interface.
\end{abstract}

\section{Introduction}

The knowledge acquisition phase of expert system development involves a knowledge engineer interview an expert and transcribe the elicited knowledge in a machine usable form. Automatic knowledge acquisition tools perform the same job of interview and knowledge transcription. These tools eliminate the possibility of errors that could be introduced by personal biases of the knowledge engineer and allow for rapid prototyping.

One of the most important automated knowledge acquisition tools is based on personal construct theory that emerged in the domain of clinical psychology in the mid '50s [3]. Boose applied personal construct theory in the development of knowledgebased systems [1]. Personal construct theory provides the basic tool to automate the structured interview technique resulting in a knowledge structure - the repertory grid.
According to personal construct theory, a person classifies the objects with which he/she interacts. These classes of objects have a direct effect on the person's behavior under any set of circumstances. Personal construct theory elicits the important classes and establishes a relationship between different objects while retaining the point of view of the person. This elicitation is achieved through a structured interview.

Before the interview, a person gives examples of important objects in his/her environment. The objects given by the interviewee are known as entities and are said to form the training set for the system. During the interview, the interviewer selects three entities from the training set at random and presents them to the interviewee. The interviewee then distinguishes between these entities such that two entities in the triad have a common property not possessed by the third. This property is called a construct. Once a construct has been elicited, the interviewee assigns a rating to each entity in the training set to reflect the extent of relevance of the construct in the description of that entity. This process is repeated many times to elicit as many constructs as possible. During each iteration, the interviewee is presented with a fresh triad of entities from the training set. The interviewee can add entities to the training set, and provide a rating for the new entities with respect to all the constructs that have been previously elicited. The interviewee can also add important constructs that may not have been possible with the random combination of entities being presented. All the ratings elicited from the interviewee are structured in the form of a matrix known as a repertory grid.

A repertory grid is a knowledge structure that stores a user's viewpoint in the domain of interest. The repertory grid provides data for approximating the probability distribution of each construct with re- 
spect to the entities in the training set. The data in the repertory grid is analyzed to quantify and display the extent of mutual relationships between entities or constructs. The extent of mutual relationship shows the degree of co-occurrence of constructs in the interviewee's point of view.

In the current interview schemes, the interviewee indicates the extent of relevance of an entity to a construct using an integer selected from a predefined rating scale. An example of such a rating scale can be a scale of 1 (completely irrelevant) to 7 (extremely relevant) with other values signifying the intermediate extent of relevance. During such interviews, we observed that even when an interviewee is familiar with the entities to a great extent, he/she is at times ambivalent about the assignment of ratings to some entities with respect to the elicited constructs. At times, the interviewee wants to assign a range to describe the ratings (for example, from "good" to "excellent"). Since the purpose of the knowledge acquisition process is to make it easy for the interviewee to express his/her viewpoint, it is obvious that there is need for a methodology that allows the expression of a range of ratings rather than a crisp value.

This led to the idea of developing an interview scheme that allows the interviewee to assign a range of values to provide the rating. Such a system should encompass the existing systems of crispvalued ratings as a special case. The addition of multivalued ratings poses a problem in the analysis of the elicited repertory grid. The post-interview analysis of the new grids must be modified to reflect the existence of a new dimension in the grid.

In this paper, we present the idea of an interval to represent the ratings of a construct with respect to an entity. We call the resultant system as a fuzzy knowledge acquisition system because of the fact that the interviewee provides an interval to describe the ratings, without a firm commitment to any particular integer even within the interval.

\section{New Interview Approach}

This section provides the details of the structured interview that elicits the ratings from a person in the form of an interval. The set up for the interview proceeds in the same manner as the conventional interview. After eliciting a construct, the system presents each entity in the training set to the interviewee and elicits his/her opinion of the entity with respect to the elicited construct in the form of a rating on a rating scale. In our system, this rating is elicited in the form of a pair signifying the lower-bound and upper-bound for the relevance of a construct in the description of an entity. The interviewee can respond in the form of an interval to indicate the belief and plausibility, or can provide a crisp rating by keeping the same value for both the upper and lower bound.

Let the rating scale be an interval between two integers 1 and $k$. Then, a rating of $[1,1]$ implies full confidence in the fact that the construct is not relevant in the description of the entity. A rating of $[k, k]$ implies full confidence that the construct is extremely relevant in the description of the entity. In a number of cases, a person may not know the extent of relevance. Such a case can be easily taken care of by a rating of $[1, k]$. Similarly, in a case in which the expert is sure that the construct is not relevant to the entity but does not know the extent, he/she can assign a rating of $[1, l](l \ll k)$. If the person has a high degree of confidence in the construct being relevant, but short of full confidence, he/she can assign a rating of $[l, k](k-l \ll k)$.

In its current implementation, our system saves the responses from the interviewee in a data base. These responses include the training set of entities, the set of constructs elicited during the interview, and the ratings of entities with respect to constructs. The data base can be later edited to reflect the changes in the interviewee's pattern of thinking. The editing is performed through the system to maintain the consistency of elicited data.

The ratings assigned by the interviewee are saved in the form of a repertory grid. The analysis of a repertory grid can provide insight into the interviewee's patterns of thinking. In the next section, we discuss the analysis of a new repertory grid.

\section{Repertory Grid Analysis}

A repertory grid provides a convenient framework to observe the extent of relevance of a construct to the entities, or vice versa, in the training set. The raw data in the repertory grid can also be analyzed to readily quantify the extent of mutual relationships between different constructs, or the extent of similarity between the entities. In this section, we will present a new analysis method suitable for the fuzzy grids and contrast its performance with the cluster analysis technique for a corresponding conventional grid. This method is adapted from the cluster analysis method investigated by Shaw [4]. 
The original repertory grids devised by Kelly were based on strict boolean values for the ratings. In boolean-valued grids, the constructs were rated to be either present or absent in the entities. The grids were extended by the researchers to multivalued ratings on a predefined scale. The ratings, however, are meant to be in the form of crisp integers. Thus, current repertory grids are limited to crisp rating values and are essentially two-dimensional structures. The new repertory grid, on the other hand, allows for the interval-valued ratings and can be abstracted as a three-dimensional structure. The third dimension is added to allow more than one rating value for each construct with respect to each entity. Representation of interval-valued ratings presents new problems in the grid analysis.

Let the repertory grid consist of ratings on $n$ elements and $m$ constructs. The rating assigned to entity $e_{i}$ with respect to the construct $c_{j}$ is denoted by $r_{i j}$ and is found at the intersection of the $i$ th column and $j$ th row of the grid. The distance between two constructs $c_{j}$ and $c_{j^{\prime}}$ is denoted by $D_{j, j^{\prime}}$ while the similarity between the same two constructs is denoted by $\sigma_{j, j^{\prime}}$. The distance in a conventional grid is given by

$$
D_{j, j^{\prime}}=\sum_{i=1}^{n}\left|r_{i j}-r_{i j^{\prime}}\right|
$$

The distance measure between each pair of constructs is used to compute the similarity $\sigma$ between two constructs using the following expression [2].

$$
\sigma_{j, j^{\prime}}=\frac{-200 \times D_{j, j^{\prime}}}{((k-1) \times n)}+100
$$

where $k$ is the maximum possible rating and $n$ is the number of entities in the training set.

The interval rating represents the extent of uncertainty in the interviewee's opinion. The fuzziness in the acquired knowledge makes it harder to analyze a grid to develop a cluster of constructs (or entities).

Our first attempt in the analysis of a fuzzy grid is motivated by providing a maximum and minimum level of confidence in the similarity matrix. This is achieved by using Expressions 1 and 2 from the conventional grid analysis by treating the pair of values representing the interval rating as separate entities. This evaluation is performed by first evaluating the difference and similarity between every pair of constructs on the basis of the lower limit in the rating interval and then, by performing the same computation on the upper limit of the interval. The analysis for the pairwise similarity between entities proceeds in an analogous manner.

Each element in the similarity matrix is in the form of an ordered pair $(u, v)$. $u$ represents the least amount of confidence in the similarity of two constructs and $v$ represents the maximum possibility of their similarity. It is important to note that both $u$ and $v$ are treated independently of the other in the computation of the cluster tree. In effect, we develop a cluster tree corresponding to the $u$ values and another tree corresponding to the $v$ values.

A cluster tree resulting from a similarity matrix is presented in Figure 1. The solid line in this figure indicates the tree derived from the least confidence in similarity (the $u$ values) while the dashed line indicates the maximum possibility of similarity (the $v$ values). The maximum possibility of similarity between some pairs of constructs might be even $100 \%$ (given by the dashed line that almost hugs the $\mathrm{x}$ axis) while the least confidence may be zero. Overall, the two clusters tend to follow the same pattern for the experiments conducted by us.

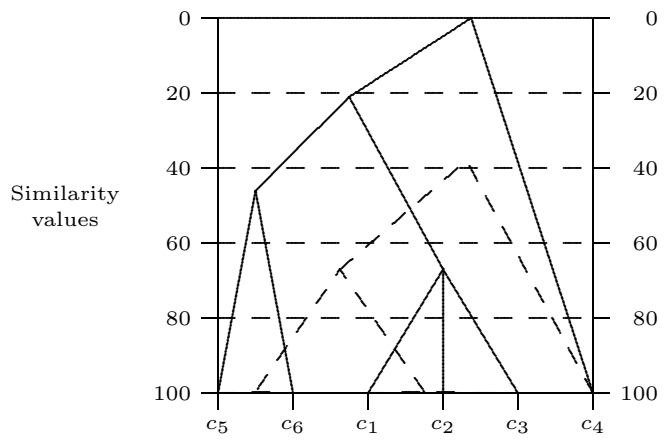

Figure 1: Cluster tree of constructs (fuzzy grid)

This first attempt at analysis is useful to develop a measure of confidence and possibility of similarity between two constructs (or entities) from an expert's viewpoint. Our future efforts will be concentrated on improving the analysis phase using the probability theory.

\section{Conclusion}

In this paper, we have presented a new user interface for an automated interview using the principles of personal construct theory. The new interface is based on allowing the expert a fuzzy expression of knowledge while doing away with the prevalent emphasis on exactness in the existing techniques. A fuzzy expression of knowledge makes the tool easy 
to use for a domain expert compared with the existing interfaces. Still, the better features as well as the results from conventional techniques are preserved. Our future efforts are focused on the improvement of the analysis phase in the entire knowledge acquisition process based on a fuzzy repertory grid.

\section{References}

[1] J. H. Boose. Personal Construct Theory and the Transfer of Human Expertise. In T. O'Shea, editor, Advances in Artificial Intelligence. Elsevier-Science Publishers B. V. (NorthHolland), 1985.

[2] A. Hart. Knowledge Acquisition for Expert Systems. McGraw-Hill, New York, NY, 1986.

[3] G. A. Kelly. The Psychology of Personal Constructs. Norton Publishers, 1955.

[4] M. L. G. Shaw and B. R. Gaines. An Interactive KnowledgeElicitation Technique Using Personal Construct Technology. In A. L. Kidd, editor, Knowledge Acquisition for Expert Systems: A Practical Handbook, pages 109-136. Plenum Press, New York, NY, 1987. 